Reprod. Nutr. Dévelop., 1986, 26 (1 B), 355-356.

\title{
Teneur en lipides du foie chez la vache en début de lactation : comparaison des différentes techniques d'estimation des triglycérides hépatiques
}

\author{
A. MAZUR, D. BAUCHART, Y. CHILLIARD, R. DIDIER $\left({ }^{*}\right)$, Y. RAYSSIGUIER
}

I.N.R.A., Theix 63122 Ceyrat, France.

(*) Laboratoire de Biologie animale,

Université Clermont II, 63170 Aubière.

Summary. This paper compares different techniques for estimating liver lipids in cows at the onset of lactation. Morphometric analysis after electron microscopic examination, and enzymatic, TLC-GLC or TLC-FID methods are discussed.

L'objectif de cette étude est de caractériser l'infiltration lipidique du foie chez la vache laitière en début de lactation par la mise en œuvre et la comparaison de différentes techniques chimiques et histologiques.

Matériel et méthodes. Quatre vaches laitières Pie-Noires ont été sacrifiées le $5^{e}$ jour et quatre autres le $56^{e}$ jour après le vêlage. Les prélèvements de foie ont été fixés immédiatement en vue d'une étude histologique, ou congelés à $-80^{\circ} \mathrm{C}$ pour des analyses biochimiques. L'étude histologique en microscopie électronique, suivie d'une analyse morphométrique, a permis de quantifier l'infiltration lipidique, exprimée en \% de la surface des hépatocytes occupés par des gouttelettes lipidiques. La conversion de la surface d'infiltration lipidique en taux de triglycérides a été effectuée selon la relation obtenue par Gaal et al. (1983) $(10,4 \%=27,6 \mathrm{mg}$ de triglycérides par gramme de tissu frais). Après extraction des lipides selon la technique de Folch, la teneur en triglycérides a été déterminée par trois méthodes différentes: 1) Une méthode enzymatique qui consiste à évaluer le glycérol après saponification alcaline des lipides et neutralisation par $\mathrm{MgSO}_{4} \quad 0,15 \mathrm{M}$. 2) Une méthode (TLC-GLC) utilisant la chromatographie sur plaque de Kieselgel (TLC), l'analyse des acides gras par chromatographie en phase gazeuse (GLC) et étalonnage interne des différentes classes de lipides séparées. 3) Une méthode (TLC-FID) couplant la séparation des lipides par chromatographie sur baguettes de quartz imprégnées de Kieselgel (TLC) et leur détection en présence d'étalon interne par ionisation de flamme (FID) sur l'analyseur latroscan (TH 10).

Résultats et discussion. Au $5^{e}$ jour après le vêlage, l'étude histologique montre une infiltration lipidique sous forme de gouttelettes qui occupent $10,2 \pm 3,0 \%$ de la surface de l'hépatocyte. Au 56 jour, les dépôts lipidiques disparaissent presque totalement $(0,9 \pm 0,4 \%)$, alors que les grains de glycogène deviennent plus abondants. Par ailleurs, des mitochondries dégénérées apparaissent plus fréquemment. Les études biochimiques confirment l'apparition d'une stéatose en début de lactation qui régresse par la suite (Reid et Collins, 1980). Celle-ci résulte essentiellement d'un dépôt de triglycérides (tabl. 1). 
Toutefois, les animaux étudiés ne manifestaient pas une infiltration lipidique particulièrement importante, la stéatose étant considérée comme pathologique pour des teneurs du foie en triglycérides supérieures à $50 \mathrm{mg} / \mathrm{g}$ de tissu frais (Reid et Roberts, 1983).

TABL. 1. - Teneur du foie en lipides chez la vache en début de lactation, déterminée par la méthode $T L C-F I D(\mathrm{mg} / \mathrm{g}$ tissu frais; moyenne \pm erreur type de 4 animaux).

\begin{tabular}{lrr}
\hline \multicolumn{1}{c}{ Jours après le vêlage } & \multicolumn{1}{c}{5} & \multicolumn{1}{c}{56} \\
\hline Esters de cholestérol & $1,4 \pm 0,2$ & $1,4 \pm 0,2$ \\
Acides gras libres & $0,7 \pm 0,1$ & $1,2 \pm 0,2$ \\
Triglycérides & $30,4 \pm 8,8$ & $4,7 \pm 0,6$ \\
Cholestérol & $0,6 \pm 0,1$ & $1,1 \pm 0,1$ \\
Phospholipides & $28,7 \pm 4,1$ & $35,0 \pm 1,3$ \\
\hline
\end{tabular}

La composition en acides gras des triglycérides, ainsi que des autres classes lipidiques du foie, n'est pas significativement différente aux deux stades étudiés. Riches en C16 : 0 (40\%) et en C18 : 1 (30\%), les triglycérides se distinguent des acides gras libres par un faible contenu en acides gras polyinsaturés. Les esters de cholestérol, comme les phospholipides, sont riches en acides gras polyinsaturés (15 à $24 \%$ ) mais diffèrent essentiellement par leurs contenus respectifs en acides gras à $16 \quad(35 \%$ contre $16 \%$ en moyenne respectivement) et 18 (40\% contre $70 \%)$ atomes de carbone.

La comparaison des 3 méthodes chimiques de dosage des triglycérides (tabl. 2) montre des corrélations très élevées, comprises entre 0,981 et 0,998; les corrélations entre la méthode morphométrique et les trois méthodes chimiques restent élevées, de 0,861 à 0,930 .

TABL. 2. - Comparaison des teneurs en triglycérides hépatiques obtenues par les différentes méthodes (mg/g tissu frais; moyenne \pm erreur type de 4 animaux).

\begin{tabular}{lrrr}
\hline \multicolumn{1}{c}{ Jours après le vêlage } & \multicolumn{2}{c}{5} & 56 \\
\hline Méthode enzymatique & $27,3 \pm 9,6$ & $5,5 \pm 1,3$ \\
TLC-GLC & $31,0 \pm 11,4$ & $5,8 \pm 2,6$ \\
TLC-FID & $30,4 \pm 8,8$ & $4,7 \pm 0,6$ \\
Analyse morphométrique & $27,0 \pm 7,9$ & $2,3 \pm 1,2$ \\
\hline
\end{tabular}

II faut toutefois souligner l'intérêt particulier du dosage par voie enzymatique ou par TLC-FID qui nécessitent un échantillon tissulaire très faible (de l'ordre de $100 \mathrm{mg}$ de poids frais) compatible avec les techniques de biopsies hépatiques.

Gaal T., Roberts C. J., Reid I. M., Dew A. M., Copp C. M., 1983. Blood composition and liver fat in post parturient dairy cows. Vet. Rec., 113, 53-54.

Reid I. M., Collins R. A., 1980. The pathology of post-parturient fatty liver in high-yielding cows. Invest. Cell Pathol., 3, 237-249.

Reid I. M., Roberts C. J., 1983. Subclinical fatty liver in dairy cows. Current research and future prospects. Irish Vet. J., 37, 104-110. 